\title{
When Irrelevant Alternatives Do Matter. The Effect of Focusing on Loan Decisions*
}

\author{
Barna Bakó $\quad$ Gábor Neszveda ${ }^{\ddagger} \quad$ Linda Dezső ${ }^{\S}$
}

August 6, 2017

\begin{abstract}
In this paper we investigate some implications of recent results about salience on loan decisions. Using the framework of focus-weighted utility we show that consumers might take out loans even when that yield them negative utility due to the focusing bias. We suggest, however, that this can be counterbalanced and consumers might be more prudent in their decisions and less likely to take out such loans when the usual fixed-installments plan is coupled with an equivalent decreasing-installments option. Moreover, we show that this is true even for loans with prepayment options or when borrowers take default into consideration. We argue that harmful loan consumption could be decreased if a policy would prescribe presentation of loan repayment schedules in a way that employs this effect.
\end{abstract}

Keywords: focus-weighted utility, loan decisions, welfare analysis, regulation

${ }^{*}$ The first author gratefully acknowledges financial support from the Hungarian National Research, Development and Innovation Office (NKFI-119930) and from the Hungarian Academy of Sciences (MTA) through the Bolyai János Research Scholarship.

${ }^{\dagger}$ Department of Microeconomics, Corvinus University of Budapest and MTA-BCE „Lendület” Strategic Interactions Research Group, Fôvám tér 8, 1093 Budapest, Hungary, tel: 0036-1-482 5165, e-mail: barna.bako@uni-corvinus.hu.

${ }^{\ddagger}$ Department of Finance, Tilburg University, Warandelaan 2, 5037 AB Tilburg, The Netherlands

${ }^{\S}$ Department of Applied Psychology, University of Vienna, Universitätsstraße 7, 1010 Wien, Austria 


\section{Introduction}

Suppose you are about to purchase a laptop, worth $\$ 1000$. A shop offers it with a loan option which lets you to pay it off in two equal installments of $\$ 500$ each. An other shop offers you the same item with two different possible ways to pay for: either of two equal-size installments of $\$ 500$ each (same as the first shop's offer), or a decreasing installments of $\$ 750$ and $\$ 250$, respectively. Assuming you would purchase the laptop at the first shop, would you buy it at the second shop if you happened to see their offer first? Based on the classical discounted utility model, rational decision-makers would consider the second shop's offer at least as good as the first's one. That is, if one would choose to purchase the item based on the first shop's offer, she would definitely purchase it when she is faced with the options offered by the second shop.

However, a growing body of evidence from laboratory and field experiments suggests that this might not necessarily be the case. Provocative examples are presented for example in Schkade and Kahneman (1998) or in Dunn et al. (2003). In these articles the authors find strong support for the observation that decision-makers when faced with multi-dimensional decisions tend to overweight few attributes of a decision relative to the others leading to counterintuitive results. ${ }^{1}$

Disproportionate weighting of attributes has been intensely researched and the notion recently was formalized for both risky decisions (Bordalo et al., 2012a) and intertemporal choices (Kôszegi and Szeidl, 2013). ${ }^{2}$ The main assumption in these models is that people tend to assign greater weight to the importance of an attribute in which their alternatives differ more. ${ }^{3}$ While the model of Kôszegi and Szeidl (2013) shows obvious similarities to the one presented in Bordalo et al. (2012a,b, 2013a,b, 2014) it assumes a weight function which is not option-specific in contrast to the option-specific characterisation suggested by Bordalo et al. and thus it is more suitable to draw welfare conclusions and regulatory implications. The model builds on a time-separable utility function where each attribute

\footnotetext{
${ }^{1}$ More related examples can be found in Huber et al. (1982), Simonson (1989), Tversky and Simonson (1993) or Roelofsma and Read (2000). For a detailed review of related experimental findings see for example Camerer et al. (2004). More recently, Bertrand et al. (2010) presented field experiment evidence about how context specific information changes the decision-maker's behaviour.

${ }^{2}$ For earlier works on this literature see Tversky (1969), Tversky and Simonson (1993), González-Vallejo et al. (1996), Roelofsma and Read (2000), González-Vallejo (2002), Scholten and Read (2010) or González-Vallejo et al. (2012).

${ }^{3}$ These approaches have been successful in explaining a range of puzzling observations in different fields of economic decisions. More specifically, the model of Bordalo and his colleagues can account for the decoy effect (Bordalo et al., 2013a), the endowment effect (Bordalo et al., 2012b) the anchoring effect (Bordalo et al., 2014), provides an explanation on how salience leads to a transformation of objective probabilities into probability weights (Bordalo et al., 2012a) and explains several puzzles associated with asset prices (Bordalo et al., 2013b). Furthermore, the model of Köszegi and Szeidl (2013) explains time-inconsistent behavior, both present bias and overcommitment to future goals at the same time, price sensitivity in health decisions (Abaluck, 2011), loan financing without budget constraints (Bertaut et al., 2009; Stango and Zinman, 2009) and lump-sum preferences compared to annuity in retirement and health decisions (Brown et al., 2008).
} 
measures the consumption in a given time period and welfare is defined as the sum of the utilities of the respective consumption. In this framework the decision-maker maximizes her focus-weighted utility based on which the utility of a time period is weighted with a focus function. This framework, however, does not specify the focus function in the presence of discounting and such its applicability is somewhat limited in cases when time is an important factor of the decision.

The main purpose of this paper is to examine the effects of focusing on loan decisions. We extend the model of Köszegi and Szeidl (2013) and make the framework more suitable for analyzing intertemporal decisions by introducing discounting and consider a more general case of the model of focusing. We analyze two different specifications of the focus function: decision-makers either focus on the nominal values or on the discounted values of the utility. We show that a decision-maker's disproportionate focus on the initial benefit a loan entails (e.g. when receiving money or a purchased good) can lead to decisions which yield negative consumption utility. ${ }^{4}$ However, as we will show in this paper this can be counterbalanced by introducing a specific alternative repayment schedule. In particular, we claim that the introduction of a decreasing-installments plan in addition to an existing fixed-installments plan makes the decision-maker less likely to take out loans which would yield her negative utility. That is, adding well designed new alternatives to the choice-set decreases the bias towards taking out harmful loans and as a consequence increases welfare. This might have important implications for policy making regarding loan consumption. We also find that even tough decreasing repayment plans have a positive effect on loan decisions they are always dominated by fixed-installment plans. This latter result is consistent with the empirical data in Cox et al. (2015) which suggest that decreasing repayment plans (e.g., equal principal repayment plan) are the least frequently chosen instruments in the loan market when other plans are also available to the consumers. Furthermore, we show that in some specific cases the introduction of an increasing-installments plan can further increase the focusing bias. This effect may contribute to the popularity of alternative mortgages, which gained a large market share both in the US and Europe (see Demyanyk and Hemert, 2011; Cox et al., 2015). One may argue that this is due to their lower initial installments, which might be especially attractive for borrowers with low income or poor credit scores, who do not have alternative options. However, empirical data do not support this explanation. The average annual household income for borrowers does not differ across mortgage types as it was reported by Cocco (2013), Amromin et al. (2011) or Cox et al. (2015). Based on the theory presented in this paper we argue that the popularity of alternative mortgages might be because of their strong focusing bias.

In what follows we present our model, derive its propositions and finally interpret our results.

\footnotetext{
${ }^{4}$ This result is in line with the empirical observation that people tend to underestimate the burden of a loan (Hoelzl et al., 2009; Akers, 2014).
} 


\section{The Model}

Let the consumption choice-set be given by a finite set $C \subset \mathbb{R}^{T+1}$, where $T>1$. Adapting the framework of Köszegi and Szeidl (2013) we assume that the consumer maximizes her focus-weighted utility given by $\bar{U}(\mathbf{c})=\sum_{t=0}^{T} g_{t} u_{t}\left(c_{t}\right)$, where $g_{t} \equiv g\left(\max _{\mathbf{c}} u_{t}\left(c_{t}\right)-\right.$ $\min _{\mathbf{c}} u_{t}\left(c_{t}\right)$ ) is a focus-weight of period (attribute) $t$ with $g(\cdot)$ a positive, strictly increasing function. However, by consuming $\mathbf{c}=\left(c_{0}, c_{1}, \ldots c_{T}\right) \in C$ the decision-maker realizes her consumption-based utility of $U(\mathbf{c})=\sum_{t=0}^{T} u_{t}\left(c_{t}\right)$.

In order to make the model more specific and relevant to intertemporal choices, we introduce discounting and consider focus-weighted utility given by $\bar{U}(\mathbf{c})=\sum_{t=0}^{T} \delta(t) g_{t} u_{t}\left(c_{t}\right)$, where $\delta(t): \mathbb{N} \rightarrow(0,1]$ is the common discount function and it is assumed to be decreasing. Furthermore, we consider consumption-based utility or personal welfare as $U(\mathbf{c})=$ $\sum_{t=0}^{T} \delta(t) u_{t}\left(c_{t}\right)$. It is important to note that with this specification of the focus-weights the decision-maker's focus is based on the nominal values rather than on discounted values of the utilities. In section 3 we will show the consequences of the model when the focus-weights are defined on the discounted values.

First we will consider the case of exponential discounting, i.e. $\delta(t)=\delta^{t}$. Then, in section 6 we will discuss the robustness of the results for more general $\delta(t)$ discount functions.

We make the following assumptions:

Assumption $1 u_{t}\left(c_{t}\right)=c_{t}$

Assumption $20 \in C$

Assumption $3(L,-x, \ldots,-x) \in C$

These assumptions are not restricting the explanatory power of the model in any relevant case. As it is shown by Kôszegi and Szeidl (2013) the model remains valid for any arbitrary functional form of the focus function. By assuming linear utility functions we follow the literature in this regard and assume that the utility function is a money metric measure. Furthermore, we assume that the loan free status-quo is always in the choice-set, that is, not taking out a loan is always an option for the decision-maker. Since the fixed-installments plan is the most typical observed loan in practice we assume that the flat plan as a possible repayment plan is always available in the choice-set.

Now, consider the following two consumption profiles: $\mathbf{c}^{\mathbf{A}}=(L,-x,-x, \ldots,-x)$ where $L, x \geq 0$ and $\mathbf{c}^{\mathbf{0}}=(0,0,0, \ldots, 0)$. One can think of $\mathbf{c}^{\mathbf{A}}$ as a loan with fixed-installments (i.e., flat plan or annuity) and $\mathbf{c}^{\mathbf{0}}$ as the loan-free status-quo. ${ }^{5}$

\footnotetext{
${ }^{5}$ Notice that we do not restrict our analysis to the case of fair loans only. Throughout the analysis a loan is considered in the most general way as a consumption profile, which can yield negative or non-negative utility to the consumer.
} 
Based on the aforementioned framework, a consumer is going to choose $\mathbf{c}^{\mathbf{A}}$ instead of $\mathbf{c}^{\mathbf{0}}$, whenever $\bar{U}\left(\mathbf{c}^{\mathbf{A}}\right) \geq \bar{U}\left(\mathbf{c}^{\mathbf{0}}\right)$, i.e when:

$$
g(L) L-\sum_{t=1}^{T} \delta^{t} g(x) x \geq 0
$$

Note, however, that consuming $\mathbf{c}^{\mathbf{A}}$ could lead to a negative consumption utility, while (1) is still satisfied. To illustrate that this might be the case, consider the following example.

Example 1 Let $T=3, \delta=0.9$ and $g_{t}=\max _{\mathbf{c}} u_{t}\left(c_{t}\right)-\min _{\mathbf{c}} u_{t}\left(c_{t}\right)$, with $\mathbf{c}^{\mathbf{A}}$ and $\mathbf{c}^{\mathbf{0}}$ as follows:

\begin{tabular}{ccccc} 
& 0. & 1. & 2. & 3. \\
\hline $\mathbf{c}^{\mathbf{A}}$ & 1000 & -600 & -600 & -600 \\
$\mathbf{c}^{\mathbf{0}}$ & 0 & 0 & 0 & 0 \\
\hline$g_{t}=\max _{\mathbf{c}} u_{t}\left(c_{t}\right)-\min _{\mathbf{c}} u_{t}\left(c_{t}\right)$ & 1000 & 600 & 600 & 600 \\
\hline
\end{tabular}

In this case the consumer chooses $\mathbf{c}^{\mathbf{A}}$, since $\bar{U}\left(\mathbf{c}^{\mathbf{A}}\right)=1000 \cdot 1000-0.9 \cdot 600 \cdot 600-0.9^{2}$. $600 \cdot 600-0.9^{3} \cdot 600 \cdot 600=121960>0$, however, $\mathbf{c}^{\mathbf{A}}$ yields a consumption-based utility of $U\left(\mathbf{c}^{\mathbf{A}}\right)=1000-0.9 \cdot 600-0.9^{2} \cdot 600-0.9^{3} \cdot 600=-463.4<0$. Hence, a consumer focusing on the closer-to-the-present attributes may be tempted to choose a consumption profile which yields a negative utility for her.

Assumption $4 x_{t}<L$ for $t=1, \ldots, T$

We assume that all installments are strictly smaller than the lump-sum value, that is, we assume that a loan is always paid back in several installments.

Definition 1 For a given c repayment plan and discount factor the fair lump-sum value $\left(L_{W}\right)$ is the value for which the consumption-based utility of the loan is zero, i.e., $u_{0}\left(L_{W}\right)+$ $\sum_{t=1}^{T} \delta^{t} u_{t}\left(c_{t}\right)=0$.

Definition 2 For a given c repayment plan and discount factor the fair focus-weighted utility lump-sum value $\left(L_{F W U}\right)$ is the value for which the focus-weighted utility of the loan is zero, i.e., $g\left(L_{F W U}\right) u_{0}\left(L_{F W U}\right)+\sum_{t=1}^{T} \delta^{t} g_{t} u_{t}\left(c_{t}\right)=0$.

Proposition 1 For a given c repayment plan and discount factor the decision-maker is always willing to accept a lower lump-sum value based on her focus-weighted utility than based on her consumption utility, i.e. $L_{F W U}<L_{W}$. 
Proof: Since $\frac{g_{t}}{g\left(L_{F W U}\right)} \in(0,1)$ we have that

$$
\sum_{t=1}^{T} \delta^{t} \frac{g_{t}}{g\left(L_{F W U}\right)} u_{t}\left(c_{t}\right)>\sum_{t=1}^{T} \delta^{t} u_{t}\left(c_{t}\right)
$$

or $L_{F W U}<L_{W}$

This proposition indicates that it is always possible to create a loan contract which seems to be beneficial for the decision-maker even though it yields negative utility for her.

Definition 3 For a given c repayment plan and discount factor the focusing bias in loan decisions $(B)$ is the difference between the fair lump-sum values, i.e., $B=L_{W}-L_{F W U}$

To examine the effects of focusing, let us introduce one more consumption profile: $\mathbf{c}^{\mathbf{B}}=$ $\left(L,-x_{1},-x_{2}, \ldots,-x_{T}\right)$, where $x_{i} \leq x_{j}$ whenever $i \geq j$ and $x_{t} \geq 0,(i, j \in\{1,2, \ldots, T\}$ and $t=1,2, \ldots, T)$. We assume that $\sum_{t=1}^{T} \delta^{t} x_{t}=\sum_{t=1}^{T} \delta^{t} x$, where $x$ refers to the installments of $\mathbf{c}^{\mathbf{A}}{ }^{6}{ }^{6}$ One can think of $\mathbf{c}^{\mathbf{B}}$ as a decreasing loan repayment plan with a present value equal as $\mathbf{c}^{\mathbf{A}}$. In this case, the consumer's maximization problem can be written as:

$$
\max _{\mathbf{c}} \bar{U}(\mathbf{c}) \quad \text { for } \quad \mathbf{c} \in\left\{\mathbf{c}^{\mathbf{0}}, \mathbf{c}^{\mathbf{A}}, \mathbf{c}^{\mathbf{B}}\right\} .
$$

Proposition 2 Introducing a decreasing loan repayment plan in addition to a flat repayment plan decreases the focus-weighted utility of the flat plan.

Proof: Let $k \equiv \min \left\{i \mid x_{i} \leq x\right\}$. If $x_{1} \geq x$ and $x_{T} \leq x$, then $k \in\{1,2, \ldots, T\}$ is well defined, as it is the case for loan repayment plans. We shall prove that:

$$
\begin{aligned}
g(L) L- & \sum_{t=1}^{T} \delta^{t} g(x) x \\
-\sum_{t=1}^{k-1} \delta^{t} g(x) x & \geq-\sum_{t=1}^{k-1} \delta^{t} g\left(x_{t}\right) x
\end{aligned}
$$

which is equivalent to

$$
\sum_{t=1}^{k-1} \delta^{t}\left[g\left(x_{t}\right)-g(x)\right] x \geq 0
$$

\footnotetext{
${ }^{6}$ Notice, that we do not restrict our attention to alternatives with the same duration as the original plan. Throughout the analysis we allow alternative repayment plans to have shorter duration than the flat plan as far as their present value is the same. In this regard, periods with no installments should be considered as periods with $x_{t}=0$.
} 
Since $g(\cdot)$ is a positive, strictly increasing function and $\delta>0$, this inequality holds if $x_{1}, \ldots, x_{k-1} \geq x$, which is the case by definition. Moreover, if $x_{1}>x$ then the (4) holds as strict inequality.

Remark 1 Notice that Proposition 2 holds not only when a decreasing installments plan is introduced, but also when any other type of loan repayment plan is added to the choice-set in addition to the flat plan. This is because in the periods when the flat installment is greater then the alternative one the actual focus will be determined by the flat plan and hence can be eliminated, while in those periods when the installment of the alternative plan is higher relative to the flat installment than the assigned focus is also going to be greater. Thus, inequality (4) holds for any type of equivalent repayment plan. Furthermore, with a similar argument one can easily show that the same is true if the loan originally is offered with an increasing-installments plan rather than with a flat plan.

Proposition 2 yields an interesting result. If the consumer's profile-set consists only of $\mathbf{c}^{\mathbf{A}}$ and $\mathbf{c}^{\mathbf{0}}$, she chooses $\mathbf{c}^{\mathbf{A}}$, whenever (1) is satisfied. Yet, if $\mathbf{c}^{\mathbf{B}}$ is part of the set as well, she may prefer $\mathbf{c}^{\mathbf{0}}$. To demonstrate this, consider the next example.

Example 2 Let $T=3, \delta=0.9$ and $g_{t}=\max _{\mathbf{c}} u_{t}\left(c_{t}\right)-\min _{\mathbf{c}} u_{t}\left(c_{t}\right)$ again and the consumption profiles as follows:

\begin{tabular}{ccccc} 
& 0. & 1. & 2. & 3. \\
\hline $\mathbf{c}^{\mathbf{A}}$ & 1000 & -600 & -600 & -600 \\
$\mathbf{c}^{\mathbf{0}}$ & 0 & 0 & 0 & 0 \\
$\mathbf{c}^{\mathbf{B}}$ & 1000 & -780 & -670 & -300 \\
\hline$g_{t}=\max _{\mathbf{c}} u_{t}\left(c_{t}\right)-\min _{\mathbf{c}} u_{t}\left(c_{t}\right)$ & 1000 & 780 & 670 & 600 \\
\hline
\end{tabular}

In this case $\bar{U}\left(\mathbf{c}^{\mathbf{A}}\right)=1000 \cdot 1000-0.9 \cdot 780 \cdot 600-0.9^{2} \cdot 670 \cdot 600-0.9^{3} \cdot 600 \cdot 600=-9260<0$, $\bar{U}\left(\mathbf{c}^{\mathbf{0}}\right)=0$ and $\bar{U}\left(\mathbf{c}^{\mathbf{B}}\right)=1000 \cdot 1000-0.9 \cdot 780 \cdot 780-0.9^{2} \cdot 670 \cdot 670-0.9^{3} \cdot 600 \cdot 300=$ $-42389<0$. Therefore the optimal choice is $\mathbf{c}^{\mathbf{0}}$.

Furthermore:

Proposition $\mathbf{3}$ If $C=\left\{\mathbf{c}^{\mathbf{0}}, \mathbf{c}^{\mathbf{A}}, \mathbf{c}^{\mathbf{B}}\right\}$, then $\mathbf{c}^{\mathbf{A}} \succ \mathbf{c}^{\mathbf{B}}$, i.e., if a flat and a decreasing plan are both available to a consumer, then the former is always preferred.

Proof: We shall prove that:

$$
g(L) L-\sum_{t=1}^{k-1} \delta^{t} g\left(x_{t}\right) x-\sum_{t=k}^{T} \delta^{t} g(x) x \geq g(L) L-\sum_{t=1}^{k-1} \delta^{t} g\left(x_{t}\right) x_{t}-\sum_{t=k}^{T} \delta^{t} g(x) x_{t}
$$


Define $y_{i} \equiv x_{i}-x$. Note that $y_{i} \geq 0$ if $i=1,2, \ldots, k-1$ and $y_{i} \leq 0$ otherwise. Thus (5) can be written as:

$$
\sum_{t=1}^{k-1} \delta^{t} g\left(x+y_{t}\right) x+\sum_{t=k}^{T} \delta^{t} g(x) x \leq \sum_{t=1}^{k-1} \delta^{t} g\left(x+y_{t}\right)\left(x+y_{t}\right)+\sum_{t=k}^{T} \delta^{t} g(x)\left(x+y_{t}\right)
$$

This simplifies to:

$$
0 \leq \sum_{t=1}^{k-1} \delta^{t} g\left(x+y_{t}\right) y_{t}+\sum_{t=k}^{T} \delta^{t} g(x) y_{t}
$$

or

$$
0 \leq \sum_{t=1}^{k-1} \delta^{t} g\left(x+y_{t}\right) y_{t}+g(x) \sum_{t=k}^{T} \delta^{t} y_{t}
$$

Since $\sum_{t=1}^{T} \delta^{t} x=\sum_{t=1}^{T} \delta^{t}\left(x+y_{t}\right)$, we have that $\sum_{t=1}^{T} \delta^{t} y_{t}=0$. Using this, (7) can be written as:

$$
0 \leq \sum_{t=1}^{k-1} \delta^{t} g\left(x+y_{t}\right) y_{t}-g(x) \sum_{t=1}^{k-1} \delta^{t} y_{t}
$$

that is

$$
0 \leq \sum_{t=1}^{k-1} \delta^{t}\left[g\left(x+y_{t}\right)-g(x)\right] y_{t}
$$

As $y_{t}>0$ for $t=1,2, \ldots, k-1$ and $g(\cdot)$ is strictly positive and increasing by definition, this inequality always holds. Moreover, the inequality is strict whenever $\exists s \in\{1,2, \ldots, T-1\}$ for which $y_{s}>0$, in other words when $\mathbf{c}^{\mathbf{A}} \neq \mathbf{c}^{\mathbf{B}}$.

Remark 2 Proposition 3 holds even if $\mathbf{c}^{\mathbf{B}}$ is not a decreasing but any other type of loan repayment plan. In this case, inequality (7) can be rewritten as $0 \leq \sum_{t \in K} \delta^{t} g\left(x+y_{t}\right) y_{t}+$ $g(x) \sum_{t \notin K} \delta^{t} y_{t}$, where $K$ is the set of indices for which the non-flat installment is higher than the respective repayment of the flat schedule.

Proposition 4 Introducing any type of repayment plan in addition to a fixed-installments plan decreases the focusing bias $B$.

Proof: According to Proposition 2 introducing an alternative equivalent repayment plan makes the fixed-installment plan less attractive and $L_{F W U}$ increases compared to the original setting. Thus, by introducing a new repayment plan the $L_{F W U}$ is increasing while the $L_{W}$ does not change. As a consequence, the focusing bias $B$ decreases. 
Proposition 5 Introducing any number of decreasing (increasing) repayment plans in addition to a fixed-install-ments plan decreases the focusing bias $B$.

Proof: Let the set of those periods in which at least one of the alternative repayment plans' relevant installment is greater than the fixed installment be $K$, i.e., $K \equiv\left\{t \mid x_{t}>\right.$ $x$ for any $\mathbf{c} \in C\}$. Since the focus weights in these periods are determined by the maximum effective installments, it follows that these are bigger that the weights effective when only the flat plan is available. However, in those periods, when the installment of any alternative plan is equal or smaller then the fixed installment the focus-weight is determined by the fixed installment. More formally, $g_{t}=g\left(\max _{\mathbf{c}} x_{t}\right)$ if $t \in K$ and $g_{t}=g(x)$ otherwise. Counterposing the two focus-weighted utility of the flat plan, we can eliminate all those periods' utilities where the focus-weight is similar, i.e., when $t \in T \backslash K$. The remaining periods are all characterized by greater focus-weights than the weights effective when only the flat plan is available, since $g_{t}(\cdot)=g\left(\max _{\mathbf{c}} x_{t}\right)>g(x)$ if $t \in K$ by definition. It follows that the focus-weighted utility of the flat plan is smaller when the flat plan is coupled with alternative repayment plans relative to the case when it is the only available repayment plan. In other words, coupling the flat plan with alternative repayment plans decreases the $L_{F W U}$ of the flat plan. Thus, if it happens that the consumer prefers the flat plan over all the alternatives then the focusing bias $B$ is decreasing following the introduction of new repayment plans.

If, however, at least one alternative dominates the flat plan the consumer would prefer to choose the loan with an alternative repayment rather than with the fixed-installments plan. Let the set of alternatives with the highest focus-weighted utility given that all alternatives are available be $D$. More formally, let $D=\{\mathbf{d} \in C \mid \mathbf{d} \succsim \mathbf{c}, \forall \mathbf{c} \in C\}$. Take an element of this set, say $\tilde{\mathbf{d}}$. We know that the $L_{F W U}$ of $\tilde{\mathbf{d}}$ is strictly greater than the $L_{F W U}$ of the fixed-installments plan when there are only these two repayment plans available in addition to the status-quo. We also know that the $L_{F W U}$ of $\tilde{\mathbf{d}}$ is getting greater as new alternatives are added to the choice-set, since the effective focus-weights are never getting smaller but potentially greater with the introduction of new repayment plans. However, since the consumer prefers $\tilde{\mathbf{d}}$ over any other repayment plans, the $L_{F W U}$ of $\tilde{\mathbf{d}}$ should be the lowest when all alternatives are available. Yet, as we have shown, this later $L_{F W U}$ is greater than the $L_{F W U}$ of the fixed-installments plan when the choice-set consist only of these two repayment plans apart from the status-quo. Consequently, the $L_{F W U}$ of $\tilde{\mathbf{d}}$ is always higher than $L_{F W U}$ of the fixed-installments plan in the original setting. Since we have chosen $\mathbf{d}$ arbitrarily it follows that the argument holds for all $\mathbf{d} \in D$. As a consequence, the introduction of new repayment plans decreases the focusing bias $B$.

Our results suggest that introducing a new repayment plan increases the $L_{F W U}$ of a flat plan. One might argue that this may possibly deter the decision-maker from taking out a loan which potentially would result in positive consumption utility. This, however, cannot be the case. Whenever the consumption utility of a loan is positive the focus-weighted 
utility of it is also positive. More formally:

Proposition 6 If $U(\mathbf{c})>0$, then $\bar{U}(\mathbf{c})>0$ also holds. To show this consider the following. The focus-weighted utility of $\mathbf{c}=\left(L,-x_{1},-x_{2}, \ldots,-x_{T}\right)$ can be written as

$$
g_{0} L-\delta g_{1} x_{1}-\delta^{2} g_{2} x_{2}-\cdots-g_{T} \delta^{T} x_{T}
$$

where $g_{t}$ is the focus-weight of period $t$. Since $x_{i}<L(i=1, \ldots, T)$, we have that $g_{0} \geq g_{t}$ for any $t=1, \ldots, T$. Thus

$g_{0} L-\delta g_{1} x_{1}-\delta^{2} g_{2} x_{2}-\cdots-g_{T} \delta^{T} x_{T} \geq g_{0} L-\delta g_{0} x_{1}-\delta^{2} g_{0} x_{2}-\cdots-g_{0} \delta^{T} x_{T}=g_{0}\left(L-\sum_{t=1}^{T} \delta^{t} x_{t}\right)$

Yet, this is always positive, since $U(c)>0$ and $g_{0}>0$.

\section{Focus-weights based on discounted values}

So far, we considered cases with focus-weights based on nominal values of the repayment plans. Let us now examine our results when focusing is based on discounted values, that is, when $g_{t} \equiv g\left(\delta^{t}\left(\max _{\mathbf{c}} u_{t}\left(c_{t}\right)-\min _{\mathbf{c}} u_{t}\left(c_{t}\right)\right)\right)$. In the following we will show that if we define focus-weights in terms of discounted values Proposition 2 to 5 still hold for decreasinginstallment plans, although not necessarily for other alternative plans such as increasinginstallment plans.

Proposition 7 When the decision-maker focuses on the discounted values of the utilities, introducing a decreasing loan repayment schedule makes a flat plan less attractive for the consumer.

Proof: In this case (4) changes to:

$$
\sum_{t=1}^{k-1} \delta^{t} g\left(\delta^{t} x_{t}\right) x-\sum_{t=1}^{k-1} \delta^{t} g\left(\delta^{t} x\right) x \geq 0
$$

Since $x_{1}, \ldots, x_{k-1} \geq x, g(\cdot)$ positive and strictly increasing, while $\delta \in(0,1]$ this inequality always holds.

Remark 3 Note that Proposition 7 holds not only for decreasing plans but for any other type of loan repayment schedule. Moreover, the same is true for an increasing-installments plan when it is coupled with new alternatives. To see this, one can use the same technic presented in Remark 1. 
Proposition 8 When the decision-maker focuses on the discounted values and $C=$ $\left\{\mathbf{c}^{\mathbf{0}}, \mathbf{c}^{\mathbf{A}}, \mathbf{c}^{\mathbf{B}}\right\}$ then $\mathbf{c}^{\mathbf{A}} \succ \mathbf{c}^{\mathbf{B}}$, i.e., if a flat and a decreasing plan are both available to a consumer, then the former is always preferred.

Proof: In this case (6) can be written as:

$$
0 \leq \sum_{t=1}^{k-1} \delta^{t} g\left(\delta^{t}\left(x+y_{t}\right)\right) y_{t}+\sum_{t=k}^{T} \delta^{t} g\left(\delta^{t} x\right) y_{t}
$$

Notice that $\sum_{t=k}^{T} \delta^{t} g\left(\delta^{t} x\right) y_{t}$ is always negative, since $y_{t}<0$ for any $t=k, \ldots, T$. That is, by replacing $\delta^{t}$ with $\delta^{k}$ for each $t=k, \ldots, T$, we have that the right-hand side of (10) is never lower than $\sum_{t=1}^{k-1} \delta^{t} g\left(\delta^{t}\left(x+y_{t}\right)\right) y_{t}+\sum_{t=k}^{T} \delta^{t} g\left(\delta^{k} x\right) y_{t}$. Hence:

$$
\sum_{t=1}^{k-1} \delta^{t} g\left(\delta^{t}\left(x+y_{t}\right)\right) y_{t}+\sum_{t=k}^{T} \delta^{t} g\left(\delta^{t} x\right) y_{t} \quad \geq \sum_{t=1}^{k-1} \delta^{t} g\left(\delta^{t}\left(x+y_{t}\right)\right) y_{t}+\sum_{t=k}^{T} \delta^{t} g\left(\delta^{k} x\right) y_{t}
$$

The right-hand side of (11) can be written as $\sum_{t=1}^{k-1} \delta^{t} g\left(\delta^{t}\left(x+y_{t}\right)\right) y_{t}+g\left(\delta^{k} x\right) \sum_{t=k}^{T} \delta^{t} y_{t}$ and since $\sum_{t=1}^{T} \delta^{t} y_{t}=0$, this equals to $\sum_{t=1}^{k-1} \delta^{t} g\left(\delta^{t}\left(x+y_{t}\right)\right) y_{t}+g\left(\delta^{k} x\right) \sum_{t=1}^{k-1}-\delta^{t} y_{t}$ or $\sum_{t=1}^{k-1} \delta^{t} g\left(\delta^{t}\left(x+y_{t}\right)\right) y_{t}-\sum_{t=1}^{k-1} \delta^{t} g\left(\delta^{k} x\right) y_{t}$ This, however, can be written as $\sum_{t=1}^{k-1} \delta^{t}\left[g\left(\delta^{t}(x+\right.\right.$ $\left.\left.\left.y_{t}\right)\right)-g\left(\delta^{k} x\right)\right] y_{t}$ As $y_{1}, \ldots, y_{k-1} \geq 0$ and $g(\cdot)$ is positive and strictly increasing, this latter expression is always non-negative, that is, (11) always holds, and as a consequence (10) is always true.

Proposition 8 is not necessarily true if $\mathbf{c}^{\mathbf{B}}$ is not a decreasing repayment plan, but for instance, an increasing one. To illustrate this consider the following example.

Example 3 Let $T=3, \delta=0.9, g_{t}=\delta^{t}\left[\max _{\mathbf{c}} u_{t}\left(c_{t}\right)-\min _{\mathbf{c}} u_{t}\left(c_{t}\right)\right]$ and the consumption profiles be as follows:

\begin{tabular}{ccccc} 
& 0. & 1. & 2. & 3. \\
\hline $\mathbf{c}^{\mathbf{A}}$ & 1000 & -600 & -600 & -600 \\
$\mathbf{c}^{\mathbf{0}}$ & 0 & 0 & 0 & 0 \\
$\mathbf{c}^{\mathbf{C}}$ & 1000 & -542 & -602 & -669 \\
\hline $\max _{\mathbf{c}} u_{t}\left(c_{t}\right)-\min _{\mathbf{c}} u_{t}\left(c_{t}\right)$ & 1000 & 600 & 602 & 669 \\
$g_{t}$ & 1000 & 540 & 487.62 & 487.7 \\
\hline
\end{tabular}

In this case $\bar{U}\left(\mathbf{c}^{\mathbf{A}}\right)=1000 \cdot 1000-0.9 \cdot 540 \cdot 600-0.9^{2} \cdot 487.62 \cdot 600-0.9^{3} \cdot 487.7 \cdot 600=258096.26$, $\bar{U}\left(\mathbf{c}^{\mathbf{0}}\right)=0$ and $\bar{U}\left(\mathbf{c}^{\mathbf{C}}\right)=1000 \cdot 1000-0.9 \cdot 540 \cdot 542-0.9^{2} \cdot 487.62 \cdot 602-0.9^{3} \cdot 487.7 \cdot 669=$ 260962.47. Therefore the optimal choice is $\mathbf{c}^{\mathbf{C}}$.

We summarize this in the following: 
Proposition 9 In case of focusing on discounted values the welfare effect of introducing a repayment plan is ambiguous, however, introducing a decreasing repayment plan still provides welfare improvement.

Remark 4 Notice that for the propositions to hold we don't need a real decreasing-installments plan in the sense that $x_{i} \geq x_{j}$ whenever $i \geq j$, we only need a repayment plan with a $k$ for which $x_{i}>x$ if $i<k$ and $x_{i} \leq x$, otherwise.

Furthermore, it is easy to show that Proposition 6 remains valid with discounted focusweights as well.

\section{The effect of focusing on loans with prepayment options}

One may wonder how the results presented in the previous sections are affected by the possibility of loan prepayments. Let us assume that borrowers do have the option to pay their loans off before their maturity. Yet, prepaying the loan, say in period $s=1, \ldots, T-1$, borrowers may face a prepayment penalty of $\eta_{s} \geq 0$, which can be either a lump-sum fee or a sum based on a percentage of the remaining balance of the loan. ${ }^{7}$ Thus, a borrower who would pay the loan off in period $s$ would be characterized with the consumption profile of $\mathbf{c}_{s}^{\text {pre }}=\left(L,-x_{1},-x_{2}, \ldots,-x_{s-1},-\sum_{t=s}^{T} \delta^{t-s} x_{t}-\eta_{s}, 0, \ldots, 0\right) .{ }^{8}$ Notice, that these prepayment options extend the choice-set with $T-1$ new elements for each consumptionprofile. In general, for a choice $\mathbf{c} \in C$, the following options are available if prepayment is possible:

\begin{tabular}{lcccccc}
$\mathrm{t}$ & 0 & 1 & 2 & $\ldots$ & $\mathrm{T}-1$ & $\mathrm{~T}$ \\
\hline $\mathbf{c}$ & $L$ & $-x_{1}$ & $-x_{2}$ & $\ldots$ & $-x_{T-1}$ & $-x_{T}$ \\
$\mathbf{c}_{\mathbf{T}-\mathbf{1}}^{\text {pre }}$ & $L$ & $-x_{1}$ & $-x_{2}$ & $\ldots$ & $-\sum_{t=T-1}^{T} \delta^{t-(T-1)} x_{t}-\eta_{T-1}$ & 0 \\
& $\vdots$ & $\vdots$ & $\vdots$ & $\vdots$ & $\vdots$ & $\vdots$ \\
$\mathbf{c}_{\mathbf{1}}^{\text {pre }}$ & $L$ & $-\sum_{t=1}^{T} \delta^{t-1} x_{t}-\eta_{1}$ & 0 & $\ldots$ & 0 & 0 \\
\hline
\end{tabular}

To see how the previous results are affected by the prepayment options available to the borrowers consider Proposition 2 first. In this case the focus-weights for each period are determined solely by the flat plan (more precisely by the present value of the remaining balance of the flat plan in the respective period plus the prepayment penalty), and the decreasing installments plan plays no role in this regard. This is because with a decreasinginstallments plan one always pays off more of the loan in the first few periods than with the flat plan, i.e. the remaining balance of the flat plan is always greater than that of

\footnotetext{
${ }^{7}$ Prepayment penalties are often used in order to protect lenders against the financial loss of paid interest over time.

${ }^{8}$ Here we assume that $\eta_{s}$ is a lump-sum fee, however, all our results remain valid with the penalty which is based on a percentage of the remaining balance of the loan.
} 
the decreasing plan. Thus, if the borrower has the option to pay her loan off in any period, inequality (4) holds. ${ }^{9}$ That is, Proposition 2 is valid even if prepayment options are available.

Proposition 3 holds as well if prepayment of the loan is an option. The reason for this is that the focus-weights are decreasing over time, as the remaining balance of the flat plan is getting smaller and smaller. Therefore, the greatest installments of the decreasing plan are overweighted, which makes the decreasing-installments plan less appealing compared to the flat plan. More formally, in Proposition 3 inequality (6) modifies to:

$$
0 \leq \sum_{t=1}^{k-1} \delta^{t} g\left(\sum_{s=t}^{T} \delta^{s-1} x+\eta_{s}\right) y_{t}+\sum_{t=k}^{T} \delta^{t} g\left(\sum_{s=k}^{T} \delta^{T-s} x+\eta_{s}\right) y_{t}
$$

Let us replace the focus-weights for each periods of $t \geq k$ with the greatest weight of this interval, i.e. with the focus-weight of the remaining balance of the flat plan in period $k$. As $y_{t} \leq 0$ if $t \geq k$ and the remaining balance of the flat plan is decreasing in each subsequent period inequality (12) holds if the following is true:

$$
0 \leq \sum_{t=1}^{k-1} \delta^{t} g\left(\sum_{s=t}^{T} \delta^{s-1} x+\eta_{s}\right) y_{t}+g\left(\sum_{s=k}^{T} \delta^{T-s} x+\eta_{s}\right) \sum_{t=k}^{T} \delta^{t} y_{t}
$$

After similar transformations as in the proof of Proposition 3, this can be rewritten as

$$
0 \leq \sum_{t=1}^{k-1} \delta^{t}\left[g\left(\sum_{s=t}^{T} \delta^{s-1} x+\eta_{s}\right)-g\left(\sum_{s=k}^{T} \delta^{T-s} x+\eta_{s}\right)\right] y_{t} .
$$

As the focus-weights are strictly decreasing and $y_{t} \geq 0$ if $t<k$ this inequality always holds. Thus, Proposition 3 remains valid even when prepayment options are available. Moreover, since Proposition 2 and 3 hold it follows that Proposition 4 and 5 are true as well. The proof of Proposition 7-9 goes along the same lines.

One result, though, which does not remain valid if prepayment options are available is Proposition 6. When the focus-weights are large enough the focus-weighted utility of a repayment plan can become negative, even though the decision-maker would benefit from taking out the loan with the respective repayment plan. As the following example illustrates a consumption profile with positive consumption-based utility can have negative focus-weighted utility when prepayment is possible.

Example 4 Let $T=3, \delta=0.8, \eta_{t}=200, g_{t}=\left[\max _{\mathbf{c}} u_{t}\left(c_{t}\right)-\min _{\mathbf{c}} u_{t}\left(c_{t}\right)\right]$ and the consumption profiles be as follows:

\footnotetext{
${ }^{9}$ Notice that, if the prepayment option is available only from the second period, i.e. $s>1$, inequality (4) holds for strict inequality. This is because, in this case (4) can be rewritten as $\delta\left[g\left(x_{1}\right)-g(x)\right] x+$ $\sum_{t=2}^{T} \delta^{t}\left[g\left(\sum_{t=2}^{T} \delta^{t-2} x+\eta_{t}\right)-g\left(\sum_{t=2}^{T} \delta^{t-2} x+\eta_{t}\right)\right] x \geq 0$. Since the first part of this inequality is strictly positive and the second part equals to zero the inequality always holds.
} 


\begin{tabular}{ccccc} 
& 0. & 1. & 2. & 3. \\
\hline $\mathbf{c}$ & 1000 & -500 & -500 & -500 \\
$\mathbf{c}_{\mathbf{1}}^{\mathbf{p r e}}$ & 1000 & -1420 & 0 & 0 \\
$\mathbf{c}_{\mathbf{2}}^{\mathbf{p}}$ & 1000 & -500 & -1100 & 0 \\
$\mathbf{c}^{\mathbf{0}}$ & 0 & 0 & 0 & 0 \\
\hline$g_{t}=\max _{\mathbf{c}} u_{t}\left(c_{t}\right)-\min _{\mathbf{c}} u_{t}\left(c_{t}\right)$ & 1000 & 1420 & 1100 & 500 \\
\hline
\end{tabular}

In this case $\bar{U}(\mathbf{c})=-48000$ and $\bar{U}\left(\mathbf{c}^{\mathbf{0}}\right)=0$. Therefore the optimal choice is $\mathbf{c}^{\mathbf{0}}$, however, taking out the loan would be beneficial, since $U(\mathbf{c})=24>0$.

Interestingly, the availability of the prepayment option has a similar reducing effect on focusing bias as coupling a repayment plan with a more decreasing plan does. As we have shown the prepayment option increases the focus-weight of each period, therefore the focusweighted utility of any repayment plan consequently decreases. Moreover, if prepayment penalties are being used, the focusing bias is going to be even smaller as the penalties further increase the focus-weights. In fact prepayment penalties could be used to totally eliminate the focusing bias the decision-maker is facing in loan decisions. If prepayment penalties in each period would be set in such a way that the focus-weighted utility of the loan would equal the consumption-based utility of it, then the focusing bias caused by the disproportionate weights would disappear. Therefore, from a welfare-perspective, we define the optimal prepayment penalty in the following way:

Definition 4 For each consumption profile the optimal prepayment penalty vector, $\eta_{\mathbf{s}}^{\mathbf{o p t}}=$ $\left(\eta_{1}^{o p t}, \eta_{2}^{o p t}, \ldots, \eta_{T-1}^{o p t}\right)$, is a vector of lump-sum fees a decision-maker has to pay in case of prepayment for which the focus-weighted utility of the consumption profile equals its consumption-based utility, i.e. is the solution of the following equation:

$$
g(L) L-\sum_{t=1}^{T} \delta^{t} g\left(\sum_{s=t}^{T} \delta^{s-1} x+\eta_{s}^{o p t}\right) x_{t}-\eta_{s}^{o p t}=L-\sum_{t=1}^{T} \delta^{t} x_{t}
$$

To determine the optimal penalties, one should know the functional form of $g(\cdot) .{ }^{10}$ This, however, is private information and not readily available for the social planner. Without this information it is difficult, if not impossible, to determine optimal prepayment penalties and one should bare in mind that poorly adjusted prepayment penalties can dissuade the decision-makers from taking out loans which would be beneficial for them. In light of this it is important to emphasize the effectiveness of the mechanism discussed in this paper. By coupling a repayment plan with a more decreasing-installments plan one can reduce the focusing bias without jeopardising the borrowing of loans with positive utility. Moreover, this mechanism is easy to implement and comes with negligible or no cost whatsoever.

\footnotetext{
${ }^{10}$ If prepayment penalties are constant over time, i.e. $\eta_{s}^{\text {opt }}=\eta$ for every $s=1, \ldots, T-1$ as it is often the case, the optimal prepayment penalty is unique, yet to determine this penalty the same information is needed as in the general case.
} 
On the other hand, these penalties may not be optimal for the lenders. From the lenders' perspective the optimal penalty would be a penalty vector which secures them against the financial loss of the paid interest in case of prepayment. Yet, these penalties, and as a matter of fact any positive penalty, would have the unintended consequence of increasing the focus-weights which may discourage decision-makers from taking out loans which may have negative impact on the lender's profit. Because of these conflicting effects a lender might be better off if borrowers would not consider the available prepayment options as feasible which is in line with the empirical findings of Lacko and Pappalardo (2010).

\section{The effect of a possible default on focusing}

Borrowers may default on their loans. This might be the result of a strategic behavior, i.e. when the borrower does have the ability to continue paying off the loan, but stops making payments. For example, in case of a mortgage if the property's value drops below the debt still owned, the borrower may prefer to default on the loan. ${ }^{11}$ Moreover, a borrower may become unable to meet her obligation of repayments as a result of an unseen event. In this section we revisit our results by assuming that decision-makers take into account the possibility of defaulting on their loans. Formally, we assume that borrowers expect an event when default is unavoidable or strategically desirable to occur with a probability $0<\zeta<1$. Let us further assume that by defaulting on the loan a borrower would face a cost of $D>0$ that includes all potential costs related to the default (e.g. legal costs, fines, etc.) above the debt still owned by the borrower. ${ }^{12}$

When default may happen with positive probability the payoffs, $z_{t}$, are calculated as the expected value of each periods' installments and the cost of default. More formally, $z_{t}=(1-\zeta)^{t-1}\left[(1-\zeta) x_{t}+\zeta\left(\sum_{l=t}^{T} \delta^{l-t} x_{l}+D\right)\right]$. To demonstrate how the possibility of a default affects the focus-weights, consider the following example, in which we assume that the decision-maker takes out a loan for three periods with a flat plan.

Example 5 Let $T=3, \delta=0.9, \zeta=0.1, D=100, x_{t}=600, g_{t}=\left[\max _{\mathbf{c}} u_{t}\left(c_{t}\right)-\right.$ $\left.\min _{\mathbf{c}} u_{t}\left(c_{t}\right)\right]$ and the consumption profiles be as follows:

\begin{tabular}{ccccc} 
& 0. & 1. & 2. & 3. \\
\hline $\mathbf{c}^{\mathbf{A}}$ & 1000 & -650 & -585 & -526.5 \\
$\mathbf{c}^{\mathbf{0}}$ & 0 & 0 & 0 & 0 \\
\hline $\max _{\mathbf{c}} u_{t}\left(c_{t}\right)-\min _{\mathbf{c}} u_{t}\left(c_{t}\right)$ & 1000 & 650 & 585 & 526.5 \\
\hline
\end{tabular}

As this example illustrates the possibility of default makes a flat plan effectively a decreasing plan. As a result, coupling a flat plan with a consumption-based utility equivalent decreasing plan, all our propositions remain valid as we will show in the followings.

\footnotetext{
${ }^{11}$ Strategic default is possible only with non-recourse loans, when the debt is secured only with the collateral, and in the case of default the lender is not allowed to collect the borrower's other assets.

${ }^{12}$ Here we are focusing on recourse loans, yet our results hold for non-recourse loans as well.
} 
Consider Proposition 2. Notice, that the decreasing plan's first period expected installment is greater than the flat plan's expected installment, since $(1-\zeta) x_{1}+$ $\zeta\left(\sum_{l=1}^{T} \delta^{l-1} x_{l}+D\right)>(1-\zeta) x+\zeta\left(\sum_{l=1}^{T} \delta^{l-1} x+D\right)$ always holds. Furthermore, since $(1-\zeta)^{T-1}\left[(1-\zeta) x_{T}+\zeta\left(x_{T}+D\right)\right]<(1-\zeta)^{T-1}[(1-\zeta) x+\zeta(x+D)]$, the opposite is true for the last period. From this follows that there is a well defined period $\left(k^{\prime} \equiv \min \left\{i \mid z_{i}^{(d)} \leq\right.\right.$ $\left.z_{i}^{(f)}\right\}$, where $z_{i}^{(j)}$ is the expected installment of plan $j$ in period $i$ ), similar to $k$ in the proof of Proposition 2, for which inequality (4) always holds, i.e. Proposition 2 remains true if one considers the possibility of default.

Proposition 3 also holds when the default has a positive probability. The proof goes along the same lines as in Proposition 3. Applying the same transformations, the proof simplifies to $\sum_{t=1}^{k^{\prime}-1} \delta^{t}\left[g\left(z_{t}^{(d)}\right)-g\left(z_{k^{\prime}}^{(f)}\right)\right] y_{t}^{\prime} \geq 0$, where $y_{t}^{\prime} \equiv z_{t}^{(d)}-z_{t}^{(f)}$, which inequality always holds. Therefore, we can conclude that the flat repayment plan is always preferred to a decreasing repayment plan even if defaulting on a loan may happen with a positive probability. Since Proposition 2 and 3 hold it follows that Proposition 4 and 5 are also true. One can use the same logic to prove Proposition 7-9.

Finally, Proposition 6 holds when the default on the loan has a positive probability if $z_{1} \leq L$, . More specifically

$$
(1-\zeta) x_{1}+\zeta\left(\sum_{t=1}^{T} \delta^{t-1} x_{t}+D\right) \leq L
$$

or

$$
\zeta D \leq L-x_{1}-\zeta\left(\sum_{t=2}^{T} \delta^{t-1} x_{t}-x_{1}\right)
$$

a rather unrestrictive condition, which is often satisfied in reality. ${ }^{13}$ That is, if the cost and/or the probability of default is sufficiently low, then a consumer will always take out a loan with positive utility since its focus-weighted utility cannot become negative even when the possibility of default is taken into account.

\section{Discussion}

So far we have assumed that decision-makers use exponential discounting, thus, we have posited that the discount rate is constant over time. However, that might not necessarily be the case. Many studies suggest that people tend to be present biased and this behavior

\footnotetext{
${ }^{13}$ For example, assuming that the probability of default of a fair loan with flat repayment plan of 10 periods is $10 \%$ it would require an unlikely high default cost of roughly eight times greater than the loan to violate this condition.
} 
may be characterized with declining rather than constant discount rates. ${ }^{14}$ The discount function with a declining discount rate is usually referred to as hyperbolic discount function. Since analytically it would be difficult to deal with the hyperbolic discount function we assume quasi-hyperbolic discounting in order to analyze the effect of focusing when decisionmakers are present biased and assume that $\delta(t)=\beta \delta^{t}$, where $\beta \in(0,1)$ is the parameter for present bias and $\delta \in(0,1]$. As it was shown by Laibson (1997) the quasi-hyperbolic discount function captures the qualitative properties of the hyperbolic discount function, yet maintains the analytical tractability of the exponential discount function. ${ }^{15}$ In this regard we can state the following:

Proposition 10 Our results remain valid in the case of quasi-hyperbolic discounting.

Proof: Using $\beta \delta^{t}$ instead of $\delta^{t}$ the relevant expressions increase $\beta$-fold. By dividing them with $\beta$ we obtain exactly the same inequalities we derived in the proofs of the previous sections.

Even though the effects of hyperbolic discounting might look similar to the focusing bias (in both cases the decision-maker weights the present consumption disproportionately, i.e. is present biased), they are very different phenomena. In order to understand this consider a hyperbolic decision-maker with no focusing bias. This decision-maker might prefer a loan with a decreasing-installments plan over a flat repayment plan since with hyperbolic discounting the periods near the present are more heavily discounted. Yet, this cannot happen with focusing bias as we have shown in Proposition 3 and Proposition 8.

Independent from the specification of the focusing function, our results suggest that introducing a decreasing repayment plan decreases the focusing bias. In other words, a loan always seems less attractive when a new decreasing plan is introduced in addition to the original plan and as a consequence the focusing bias $B=L_{W}-L_{F W U}$ decreases. Interestingly, the presence of the decreasing repayment plan affects only the focusing bias, but not the decision-maker's optimal choice in the sense that if the decision-maker chooses to take out the loan she will prefer the flat plan over the decreasing one. These results remain valid even if borrowers have the possibility to prepay their loans or when they take the default choice into account. Importantly, under reasonable assumptions the decrease of the focusing bias caused by the introduction of new decreasing repayment plans cannot deter any decision-maker from taking out a loan which delivers them positive consumption utility, it only affects harmful loan consumptions.

From a policy standpoint, one may wonder what the practical consequences of the existence of focusing bias are. In this regard our results indicate that lenders may have a strong, intended or unintended, influence on borrowers' decisions just by offering them the loans with specific repayment plans. As we have shown, a loan presented with a repayment plan featured with great focusing bias may incentivize consumption of the loan

\footnotetext{
${ }^{14}$ See e.g. Loewenstein and Prelec (1992), Camerer et al. (2004), Thaler (2005), Berns et al. (2007).

${ }^{15}$ For more on quasi-hyperbolic discounting see Laibson (1997).
} 
even if that may result in negative utility. This, however, might be counterbalanced by the mechanism presented in this paper. Based on our results we conjecture that adding a decreasing-installments plan to the choice-set will make decision-makers less likely to take out loans which yield them negative utility without affecting their attitude towards the ones with positive utility. Yet, we argue that people would still prefer to choose the fixed-installments plan from this extended choice-set. Moreover, we claim that by exploiting the aforementioned effect of focus, people might make more deliberate loan decisions. If banks, for example, would present a loan in fixed- and decreasing-installments options, they could end up getting more prudent decisions from their clients. This obviously boils down to policy making. Namely, a policy could prescribe that financial institutions present a loan repayment schedule also in a decreasing-installment option, and not only in a fixedor increasing-installment one. The induced focus on the decreasing-installments plan could dampen the increased focus on getting the loan, thereby discouraging decision-makers from taking out loans which might yield them negative utility. This may be especially important in the case of loans with increasing-intallments plans (e.g. mortgages with initial 'teaser' rates), since these instruments could generate the highest focusing bias, and as a consequence, may motivate harmful loan consumption the most.

\section{References}

Abaluck, J. (2011), 'What Would We Eat if We Knew More: The Implications of a LargeScale Change in Nutrition Labeling', Working Paper, MIT, 2011

Akers, E. J. and Chingos, M. M. (2014), 'Are college students borrowing blindly?', Brown Center on Education Policy at Brookings.

Amromin, G.; Huang, J.; Sialm, C. and Zhong, E. (2011), 'Complex mortgages', NBER Working Paper 17315

Berns, G.S., Laibson, D. and Loewenstein, G. (2007). 'Intertemporal choice - toward an integrative framework', Tends in cognitive sciences 11(11), 482-488.

Bertaut, C. C.; Haliassos, M. and Reiter, M. (2009), 'Credit Card Debt Puzzles and Debt Revolvers for Self Control', Review of Finance, 13,(4) 657-692.

Bertrand, M.; Karlan, D.; Mullainathan, S.; Shafir, E. and Zinman, J. (2010), 'What's advertising content worth? Evidence from a consumer credit marketing field experiment', The Quarterly Journal of Economics, 125(1), 263-306.

Bordalo, P.; Gennaioli, N. and Shleifer, A. (2012a), 'Salience Theory of Choice Under Risk', The Quarterly Journal of Economics, 127(3), 1243-1285.

Bordalo, P.; Gennaioli, N. and Shleifer, A. (2012b), 'Salience in Experimental Tests of the Endowment Effect', American Economic Review, 102(3), 47-52. 
Bordalo, P.; Gennaioli, N. and Shleifer, A. (2013a), 'Salience and Consumer Choice', Journal of Political Economy, 121(5), 803-843.

Bordalo, P.; Gennaioli, N. and Shleifer, A. (2013b), 'Salience and Asset Prices', American Economic Review, Papers \& Proceedings, 103(3), 623-628.

Bordalo, P.; Gennaioli, N. and Shleifer, A. (2014), 'Salience Theory of Judicial Decisions', The Journal of Legal Studies, 102(3), 47-52.

Brown, J. R.; Casey, M. D. and Mitchell, O. S. (2008), 'Who Values the Social Security Annuity? New Evidence on the Annuity Puzzle', NBER Working Paper No. 13800.

Camerer, C.; Loewenstein, G. and Rabin, M., eds (2004), Advances in Behavioral Economics, Princeton University Press.

Cocco, J. F. (2013), 'Evidence on the Benefits of Alternative Mortgage Products', The Journal of Finance, 68, 1663-1690.

Cox, R. H. G. M.; Brounen, D. and Neuteboom, P. (2015), 'Financial Literacy, Risk Aversion and Choice of Mortgage Type by Households', Journal of Real Estate Finance and Economics, 50(1), 74-112.

Demyanyk, Y. and Hemert, O. V. (2011), 'Understanding the subprime mortgage crisis', The Review of Financial Studies, 24(6), 1848-1880.

Dunn, E. W.; Wilson, T. D. and Gilbert, D. T. (2003). 'Location, location, location: the misprediction of satisfaction in housing lotteries'. Personality and Social Psychology Bulletin, 29(11), 1421-32.

Gathergood, J. and Weber, J. (2017), 'Financial Literacy, Present Bias and Alternative Mortgage Products', Journal of Banking 83 Finance, 78, 58-83.

González-Vallejo, C.; Bonazzi, A. and Saphiro, A. J. (1996), 'Effects of Vague Probabilities and of Vague Payoffs on Preference: A Model Comparison Analysis', Journal of Mathematical Psychology, 40, 130-140.

González-Vallejo, C. (2002), 'Making Trade-Offs: A Probabilistic and Context-Sensitive Model of Choice Behavior', Psychological Review, 109(1), 137-155.

González-Vallejo, C.; Harman, J. L.; Mullet, E. and Muñoz Sastre, M. T. (2012), 'An examination of the proportional difference model to describe and predict health decisions', Organizational Behavior and Human Decision Processes, 118(1), 82-97.

Hoelzl, E.; Pollai, M. and Kamleitner, B. (2009), 'Experience, prediction and recollection of loan burden', Journal of Economic Psychology, 30(3), 446-454. 
Huber, J.; Payne, J. W. and Puto, C. (1982), 'Adding asymmetrically dominated alternatives: violations of regularity and similarity hypo thesis', Journal of Consumer Research, 9(1), 90-98.

Kőszegi, B. and Szeidl, Á. (2013), 'A model of focusing in economic choice', The Quarterly Journal of Economics, 128(1), 53-104.

Lacko, J. and Pappalardo, J. (2010), 'The Failure and Promise of Mandated Consumer Mortgage Disclosures: Evidence from Qualitative Interviews and a Controlled Experiment with Mortgage Borrowers', The American Economic Review, 100(2), 516-521.

Laibson, D. (1997), 'Golden Eggs and Hyperbolic Discounting', The Quarterly Journal of Economics, 112(2), 443-477.

Loewenstein, G and Prelec, D. (1992). 'Anomalies in intertemporal choice: Evidence and interpretation', Quarterly Journal of Economics 107(2), 573-597.

Mayer, Ch.; Pence, K. and Sherlund, S. M. (2009), 'The Rise in Mortgage Defaults', Journal of Economic Perspectives, 23(1), 27-50.

Roelofsma, P. H. M. P. and Read, D. (2000), 'Intransitive Intertemporal Choice', Journal of Behavioral Decision Making, 13(2), 161-177.

Schkade, D. A. and Kahneman, D. (1998), 'Does Living in California Make People Happy? A Focusing Illusion in Judgments of Life Satisfaction', Psychological Science, 9(5), 340346.

Scholten, M. and Read, D. (2010), 'The Psychology of Intertemporal Tradeoffs', Psychological Review, 117(3), 925-944.

Simonson, I. (1989),'Choice based on reasons: the case of attraction and compromise effects', Journal of Consumer Research, 16(2), 158-174.

Stango, V. and Zinman, J. (2009), 'What Do Consumers Really Pay on Their Checking and Credit Card Accounts? Explicit, Implicit, and Avoidable Costs', American Economic Review Papers and Proceedings, 99(2), 424-429.

Thaler, R. (2005), Advances in Behavioral Economics, Russel Sage Foundation.

Tversky, A. (1969),'Intransitivity of Preferences', Psychological Review, 76(1), 31-48.

Tversky, A. and Simonson, I. (1993), 'Context-Dependent Preferences', Management Science, 39(10), 1179-1189. 